\title{
Vibrational Photoacoustic Microscopy for Depth-resolved Bond-selective Imaging of Tissues and Organisms
}

\author{
Han-Wei Wang ${ }^{1}$, Ning Chai ${ }^{2}$, Song $\mathrm{Hu}^{3}$, Wei Dou ${ }^{4}$, David Umulis ${ }^{1,4}$, Lihong V. Wang ${ }^{3}$, Michael Sturek ${ }^{1,5}$, \\ Robert Lucht' ${ }^{2}$ Ji-Xin Cheng ${ }^{1,6^{*}}$ \\ ${ }^{1}$ Weldon School of Biomedical Engineering, ${ }^{2}$ School of Mechanical Engineering, ${ }^{4}$ Department of Agricultural and Biological Engineering, \\ ${ }^{6}$ Department of Chemistry, Purdue University, West Lafayette, IN 47907. ${ }^{3}$ Department of Biomedical Engineering, Washington University in St. \\ Louis, St. Louis, MO 63130. 'Department of Cellular \& Integrative Physiology, Indiana University School of Medicine, Indianapolis, IN 46202. \\ *To whom correspondence should be addressed.E-mail:jcheng@purdue.edu
}

\begin{abstract}
We realize vibrational photoacoustic microscopy (VPA) using molecular excitation of overtone vibration and acoustic detection of the resultant pressure transients. We demonstrate 3-D VPA imaging of lipid-rich atherosclerotic plaques and lipid bodies in living Drosophila larvae by exciting $2^{\text {nd }}$ overtone of the $\mathrm{CH}$ bond stretch around $8300 \mathrm{~cm}^{-1}$. Depth-resolved spectral analysis and a penetration depth of several $\mathrm{mm}$ are available.

(C)2010 Optical Society of America

OCIS codes: (170.3880) Medical and biological imaging; (170.5120) Photoacoustic imaging.
\end{abstract}

Signals from inherent molecular vibrations provide a key approach to detecting specific molecules in cells, tissues and materials. Vibrational microscopes based on spontaneous Raman scattering and infrared absorption have been widely used for chemical imaging of unstained samples. With a 3-D spatial resolution, nonlinear vibrational microscopy based on coherent anti-Stokes Raman scattering (CARS) [1] and stimulated Raman scattering (SRS) [2] has shed new light on lipid biology. Employing ballistic photons, however, the coherent Raman scattering methods have a tissue penetration depth of c.a. $100 \mu \mathrm{m}$, which limits its potential for deep tissue imaging in clinical settings. For instance, we had to employ sectional slices of arteries to visualize the atherosclerotic plaques by CARS [3].

Here, we report a new method termed Vibrational photoacoustic (VPA) microscopy that permits 3-D vibrational imaging of tissues with a field of view and a penetration depth both in the $\mathrm{mm}$ scale. Our method is based on excitation of molecular overtone vibration and acoustic detection of the resultant pressure waves in the tissue. The frequency of an overtone band is described by $\bar{v}=\bar{v}_{1} n-\chi \bar{v}_{1}\left(n+n^{2}\right)$, where $\bar{v}_{1}$ is the frequency of the fundamental vibration, $\chi$ is the anharmonicity, and $n=2,3, \ldots$ represent the first, second, and so on, overtones. Overtone absorption is an essential principle in near-infrared (NIR) spectroscopic approach. Molecular spectra in chemical and biological samples can be unraveled according to radiation signals representing the overall overtone absorption and the elastic scattering in a sample. The bulk measurement of absorbance or reflectance, however, obscures depth information. The elastic scattering further compromises the 3-D imaging potential of NIR spectroscopy. Notably, most of the $2^{\text {nd }}$ overtone frequencies of molecules of interest are located in the NIR region from 700 to $1300 \mathrm{~nm}$, where the background tissue is minimally absorbing. Within this spectral region, overtone vibrational absorption provides opportunities to generate a chemically selective photoacoustic (PA) transient in a biological structure. The PA effect takes place when laser radiation is absorbed by a sample, thus induces local thermal elastic expansion and generates a pressure transient that propagates as an acoustic wave. The pressure transients encode the spatial information of absorbers on which the image reconstruction is grounded. The PA signal renders the mapping vessel plexuses or melanin owing to their strong electronic absorption in the visible region [4]. Other than that, labels such as dyes and nanoparticles were used as contrast agents for probing specific targets [4].

To explore VPA imaging based on overtone absorption of molecules as the contrast mechanism, we used 5nanosecond pulse trains in the NIR region generated by an Nd:YAG pumped optical parametric oscillator laser system. Radiation beam(s) were weakly focused on a microscope platform. Ultrasound transients are collected via a focused-type transducer and through a preamplifier and a signal receiver [Fig. 1(a)]. The envelope of each PA transient amplitude is retrieved for further signal analysis and image reconstruction.

To generate the PA signal from overtone excitation of $\mathrm{CH}$ bond, we first applied butanal, a $\mathrm{CH}$-rich liquid, in a glass sample tube. The VPA spectrum of $2^{\text {nd }}$ overtone absorption of $\mathrm{CH}$ vibration is peaked around $8400 \mathrm{~cm}^{-1}$, corresponding to $1190 \mathrm{~nm}$ [Fig. 1(b)]. Detection sensitivity of the VPA signal was tested using cholesterol in chloroform solution. We were able to obtain a clean VPA spectrum from $25 \mathrm{mM}$ cholesterol (data not shown). Applying the VPA spectroscopy to biologically significant samples, our spectroscopic results [Fig. 1(c)] show that $\mathrm{CH}$-rich samples produce a strong VPA signal around $1200 \mathrm{~nm}$ due to the $2^{\text {nd }}$ overtone absorption of $\mathrm{CH}$ vibration. Specifically, at $1215 \mathrm{~nm}$ the VPA signal from adipose tissues is over 7 times higher than that from blood and over 5 times higher than that from collagen. In addition, the VPA signal from the $1^{\text {st }}$ overtone absorption of $\mathrm{OH}$ is located 


\section{JTuG3.pdf}

around $1400 \mathrm{~nm}$, and the signal from the $2^{\text {nd }}$ overtone absorption of $\mathrm{NH}$ is detectable around $950 \mathrm{~nm}$. Penetration depth in a biological sample phantom made of semi-opaque collagen matrices is about $7 \mathrm{~mm}\left(\mathrm{e}^{-1}\right.$ signal level).

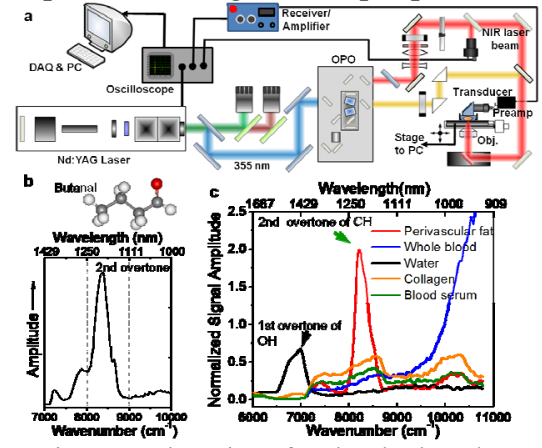

Fig. 1. PA detection of molecules based on overtone absorption.

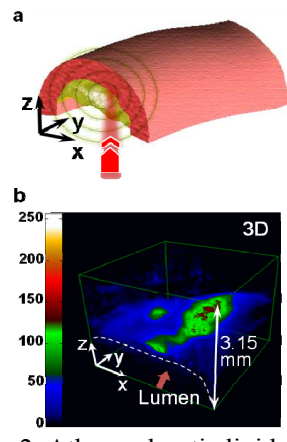

Fig. 2. Atherosclerotic lipid deposition mapped by VPA microscopy.

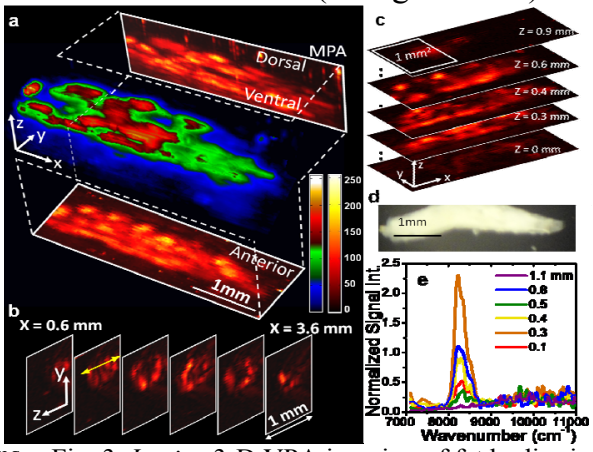

Fig. 3. In vivo 3-D VPA imaging of fat bodies in a $3^{\text {rd }}$-instar larva of Drosophila melanogaster.

To perform 3-D VPA imaging, we incorporated an XY translational stage into the system for sample scanning. The amplitude information in the time-of-flight of a pressure transient represents the locations of absorbers in the depth $(Z)$ direction. The spatial resolution was measured using an oil-filled microfluidic channel. The axial resolution is c.a. $130 \mu \mathrm{m}$ and can be improved using a transducer at a higher frequency. The lateral resolution produced by the focusing lens or objective is available from c.a. $5 \mu \mathrm{m}$.

For biomedical applications, we have performed 3-D VPA imaging of lipid-rich atherosclerotic plaques. Carotid arteries were harvested from Ossabaw pigs having metabolic syndromes and profound atherosclerosis. Spectroscopic analysis and 3-D imaging were conducted from the luminal side of the artery [(Fig. 2(a)]. We found that VPA spectroscopy allows sensing different levels of lipid accumulation at different sites of atheromatous arterial walls. According to the VPA spectra of the lipid depositions in atheromatous arterial walls, we used the radiation at $1195 \mathrm{~nm}$ for 3-D VPA imaging of atherosclerotic lipid deposition with optimal vibrational contrast from the lipid depositions. The VPA imaging can visualize milieus of atherosclerotic lipid accumulation in arterial walls, such as a confluent lipid core in an atheromatous artery [Fig. 2(c)], a scattered lipid deposition in an arterial wall, and the formation of mild fatty streaks in early atheroma. We were able to detect a strong VPA signal from lipids located at $1.5 \mathrm{~mm}$ below the lumen.

We have further applied the VPA microscope to map fat bodies in the entire $3^{\text {rd }}$-instar larvae of living Drosophila melanogaster utilizing the VPA signal of overtone absorption of $\mathrm{CH}$ bond stretch [Fig. 3(a)]. The projection and sectional images elucidate the distribution of lipid storage along the anterior-posterior and the ventral-dorsal axis [Fig. 3(b) and Fig. 3(c)]. The microphotography of the larva is shown in Fig. 3(d). We also performed depth-resolved spectra at certain points. Fig. 3(e) exemplifies the spectral analysis corresponding to the locations along the yellow arrow in Fig. 3b. It is promising for use in depth-resolved compositional analysis. Pulse energy of the radiation in our experiments was under $50 \mu \mathrm{J}$. No tissue damage, e.g. plasma formation was observed.

In summary, our work reported the VPA spectra of biological molecules and tissue components and demonstrated 3-D molecular imaging by excitation of the $2^{\text {nd }}$ overtone of $\mathrm{CH}$ bond stretch. In addition, the depthresolved spectra show the potential of providing compositional information at each depth of a sample. Such information is essential for diagnosis of diseases, such as lesion stages in atherosclerosis, where locations of compositions in depth elucidate the risk of plaque rupture [5]. We envision that the method opens up exciting opportunities for non-invasive, high resolution, intravital imaging of lipid-related disorders via quantitation of lipid deposition. Our method is also applicable to other molecules based on the $\mathrm{NH}$ and $\mathrm{OH}$ vibrations. Though the imaging speed is limited by the low repetition rate laser used in our work, the speed can be significantly improved using a laser at kilo-hertz repetition rate for excitation, using a high-speed signal digitizer for signal recording, and/or using a laser scanning scheme on a PA microscope.

\section{References}

[1] A. Zumbusch, G.R. Holtom, X.S. Xie. Three-dimensional vibrational imaging by coherent anti-Stokes Raman scattering. Phys. Rev. Lett. 1999;82:4142-4145.

[2] C.W. Freudiger, W. Min, B.G. Saar, S. Lu, G.R. Holtom, C. He, J.C. Tsai, J.X. Kang, X.S. Xie. Label-Free Biomedical Imaging with High Sensitivity by Stimulated Raman Scattering Microscopy. Science. 2008;322:1857-1861.

[3] H.-W. Wang, I.M. Langohr, M. Sturek, J.-X. Cheng. Imaging and quantitative analysis of atherosclerotic lesions by CARS-based multimodal nonlinear optical microscopy. Arterioscler. Thromb. Vasc. Biol. 2009;29:1342-1348.

[4] C. Kim, C. Favazza, L.V. Wang. In Vivo Photoacoustic Tomography of Chemicals: High-Resolution Functional and Molecular Optical Imaging at New Depths. Chemical Reviews. 2010;110:2756-2782.

[5] R. Virmani, A.P. Burke, A. Farb, F.D. Kolodgie. Pathology of the vulnerable plaque. J. Am. Coll. Cardiol. 2006;47:C13-18. 